\title{
Aerobic and anaerobic microbiology of axillary hidradenitis suppurativa
}

\author{
I. BROOK and EDITH H. FRAZIER
}

Departments of Pediatrics and Infectious Diseases, Navy Hospital, Bethesda, MD, USA

\begin{abstract}
A retrospective review of the microbiological and clinical data of 17 specimens obtained from axillary hidradenitis suppurativa (HS) over a period of 6 years was undertaken to study the aerobic and anaerobic microbiology of this condition. A total of 42 bacterial isolates (2.5 per specimen) were obtained, 12 aerobic or facultative ( 0.7 per specimen) and 30 anaerobic or micro-aerophilic (1.8 per specimen). Aerobic and facultative bacteria only were isolated in six (35\%) cases, anaerobic bacteria only in seven $(41 \%)$ and mixed aerobic and anaerobic bacteria in four (24\%). The predominant aerobic bacteria were Staphylococcus aureus (six isolates), Streptococcus pyogenes (three) and Pseudomonas aeruginosa (two). The most frequently isolated anaerobes were Peptostreptococcus spp. (10), Prevotella spp. (seven), micro-aerophilic streptococci (four), Fusobacterium spp. (three) and Bacteroides spp. sensu stricto (three). This study highlights the polymicrobial nature and predominance of anaerobic bacteria in axillary HS and the need for antimicrobial thereby to reflect this.
\end{abstract}

\section{Introduction}

Hidradenitis suppurativa (HS) is recurrent inflammation of the apocrine sweat glands, particularly those of the axilla. It can result in obstruction and rupture of the duct and secondary infection. The lesions generally drain spontaneously, with formation of multiple sinus tracts and with hypertrophic scarring.

Staphylococcus aureus was originally considered to be the most common pathogen [1]. Although anaerobic bacteria have been described in several case reports of HS [2-4], as well as in several series of axillary abscesses [4, 5], and perineal HS [6], not all previous reports adequately investigated the role of anaerobic bacteria by using appropriate methods for recovery.

This report describes a retrospective study of 6 years' experience of the isolation of aerobic and anaerobic bacteria from cases of HS in a military hospital.

\section{Materials and methods}

Between June 1984 and June 1990, 17 specimens from patients with axillary HS that were submitted to the

Received 8 Feb. 1998; revised version accepted 21 May 1998.

Corresponding author: Professor I. Brook, POB 70412, Chevy Chase, MD 20889 USA. microbiology laboratory for the isolation of aerobic and anaerobic bacteria were culture positive. A further three specimens did not show any bacterial growth. The patients' clinical and microbiological data were reviewed. Systemic antimicrobial therapy was given to seven patients during the 2 weeks before sample collection. These antimicrobial agents included a $\beta$ lactamase-resistant penicillin (4), cefazolin (2), and erythromycin (1). There were insufficient data and follow-up records to allow assessment of the subsequent management of these patients following culture collection, and to document their subsequent long-term outcome.

Eleven of the patients were male. The patients' ages ranged between 23 and 67 years (mean age 47). For specimen collection, the site was first scrubbed with povidone-iodine. Specimens were obtained from the abscesses by direct percutaneous needle aspiration of purulent contents or during surgical drainage. The pus was collected into a syringe (in 10 cases) that was immediately sealed and transported to the laboratory within 30 min or by a swab (in seven cases) that was dipped in the pus and transferred to anaerobic transport medium (Port-A-Cul, BBL Microbiological Systems, Cockeysville, MD, USA) and generally inoculated within $2 \mathrm{~h}$ of collection.

Sheep blood (5\%), chocolate and MacConkey agar plates were inoculated for the isolation of aerobic organisms. The plates were incubated at $37^{\circ} \mathrm{C}$ aero- 
bically (MacConkey agar) and in air with $\left(_{2} \quad 5 \%\right.$ (blood and chocolate agars) and examined at 24 and $48 \mathrm{~h}$. To optimise the isolation of anaerobes, specimens were plated on to pre-reduced vitamin $\mathrm{K}_{1}$ enriched Brucella blood agar, anaerobic blood agar plates containing kanamycin and vancomycin, and anaerobic blood agar plates containing colistin and nalidixic acid, and then inoculated into enriched thioglycollate broth. The plated media were incubated in GasPak jars (BBL Microbiology Systems) and examined at 48,96 and $120 \mathrm{~h}$. The thioglycollate broth was incubated for 14 days. Anaerobic and aerobic bacteria were identified as described previously $[7,8]$.

\section{Results}

A total of 42 isolates ( 2.5 per specimen) was obtained, 12 aerobic or facultative $(0.7$ per specimen $)$ and 30 anaerobic or micro-aerophilic (1.8 per specimen). Aerobic or facultative bacteria only were present in six $(35 \%)$ specimens, anaerobic or micro-aerophilic bacteria only in seven (41\%) and mixed aerobic and anaerobic bacteria were present in four $(24 \%)$.

The predominant aerobic species were $S$. aureus (six isolates), Streptococcus pyogenes (three) and Pseudomonas aeruginosa (two) (Table 1). The most frequently isolated anaerobes were Peptostreptococcus spp. (10), Prevotella spp. (seven), micro-aerophilic streptococci (four), Fusubacterium spp. (three) and Bacteroides spp. sensu stricto (three).

The number of isolates per specimen varied from one to five. A single isolate was obtained from six specimens, $S$. aureus from four Str. pyogenes from one and Pr. intermedia from one.

Foul-smelling discharge from the lesions was noted in

Table 1. Bacteria isolated from 17 hidradenitis suppurativa specimens

\begin{tabular}{llll}
\hline Aerobic bacteria & \multicolumn{2}{c}{$\begin{array}{c}\text { Anaerobic and micro- } \\
\text { aerophilic bacteria }\end{array}$} \\
\hline S. aureus & 6 & Pstr. magnus & 1 \\
S. epidermidis & 1 & Pstr. prevotii & 3 \\
Str. pyogenes & 3 & Pstr. asaccharolyticus & 2 \\
P. aeruginosa & 2 & Peptostreptococcus spp. & 4 \\
& & Gemella morbilliorum & 2 \\
& & Str. intermidius & 1 \\
& & Micro-aerophilic streptococci & 1 \\
& & F. nucleatum & 2 \\
& & F. varium & 1 \\
& & B. fragilis & 2 \\
& & B. ovatus & 1 \\
& & B. urealyticus & 1 \\
& & Pr. oris-buccae & 2 \\
& & Pr. melaninogenica & 3 \\
& & Pr. intermedia & 2 \\
Total & & Porphyromonas asaccharolytica & 2 \\
& & Total & 30 \\
\hline
\end{tabular}

nine cases; anaerobic bacteria were recovered from all these. No association was noted between any other clinical feature and the microbiological findings.

All patients were treated with antimicrobial agents and six had surgical drainage. The antimicrobial agents prescribed were clindamycin (six cases), a $\beta$-lactamase-resistant penicillin (five), cefoxitin (four), gentamicin (two), erythromycin (two) and metronidazole (two). Fourteen patients were followed up for at least 2 months. The patients' clinical response was generally slow; however, 11 recovered. The infection persisted in three cases for at least another 11 months. $P$. aeruginosa was isolated from two of these patients, in one instance with Pstr. prevotii, and in another $S$. aureus and $B$. fragilis were isolated.

\section{Discussion}

This study highlights the variety of aerobic and anaerobic bacteria isolated from patients with HS lesions. Although $S$. aureus was isolated from about a third of these patients, other aerobic bacteria such as Str. pyogenes and $P$. aeruginosa were also isolated. Anaerobic bacteria alone or in combination with aerobic organisms were isolated from about two-thirds of patients.

The anaerobes isolated from the patients are part of the flora of the oropharynx (Prevotella spp., Fusobacterium spp., Peptostreptococcus spp. and microaerophilic streptococci), gastrointestinal tract (Bacteroides spp., Peptostreptococcus spp.) [9] and skin (Peptostreptococcus spp.) and presumably reached the HS lesions from these sites. Similar organisms have been isolated from subcutaneous abscesses $[5,10]$ and wounds [10] and generally predominate in sites close to the oropharynx and rectal area.

The polymicrobial nature of HS is also highlighted in this report; the average number of isolates per specimen was 2.5. Although the pathogenic role of all bacterial isolates has not been established, the synergic character of polymicrobial infections has been well established [11-13]. Several hypotheses have been proposed to explain this. It may be partly or wholly due to mutual protection from phagocytosis and intracellular killing [14], production of essential growth factors [15], or lowering of oxidation reduction potentials in host tissues [16].

Management of HS is difficult and involves antimicrobial therapy, and moist heat locally to establish drainage in the initial phases of the infection. Surgical drainage is employed in the management of large abscesses. The findings of the present study support the reports of Leach et al. of axillary HS [4] and Highet et al. [6] of perineal HS, who also isolated anaerobic bacteria of oropharyngeal [4] and colonic origin [6]. 
This study emphasises the importance of obtaining good specimens for the isolation of aerobic as well as anaerobic bacteria from HS. The initial Gram's stain results may guide the clinician in selecting appropriate empiric antimicrobial therapy. However, the final choice of agents should be determined by the isolation of specific organisms, aerobes and anaerobes, and the results of sensitivity testing.

Surgical drainage is required in large HS abscesses, as the environment of an abscess inhibits the activity of many antimicrobial agents. The abscess capsule, the low $\mathrm{pH}$ and the presence of binding proteins or inactivating enzymes (such as $\beta$-lactamase) may impair the activity of many antimicrobial agents especially aminoglycosides. However, the administration of systemic antimicrobial agents is nonetheless indicated. Antimicrobial therapy for mixed infections due to aerobic and anaerobic bacteria requires the administration of agents effective against both aerobic and anaerobic bacterial cases of infection [17, 18].

Initial empiric antimicrobial therapy should be effective against $S$. aureus as well as other potential pathogens. Antimicrobial agents active against $S$. aureus and anaerobic bacteria include clindamycin, imipenem, cefoxitin and $\beta$-lactamase inhibitor and penicillin combinations, and metronidazole with $\beta$ lactamase-resistant penicillin. Cefoxitin and imipenem also provide coverage against Enterobacteriaceae. However, agents active against Enterobacteriaceae (i.e., aminoglycosides, quinolones, fourth-generation cephalosporins) should be added when treating infections involving these bacteria.

We acknowledge the efforts of the staff of the clinical microbiology laboratories and the clinical wards at the Naval Hospital and the secretarial assistance of Joanie Pietrafitta. The opinions and assertions contained herein reflect those of the authors and do not necessarily reflect the views of the Navy Hospital, or the Naval Service at large.

\section{References}

1. Knaysi GA, Cosman B, Crikelair GF. Hidradenitis suppurativa. JAMA 1968; 203: 19-22.

2. Brenner DE, Lookingbill DP. Anaerobic microorganisms in chronic suppurative hidradenitis (letter). Lancet 1980;2: 921-292.

3. Beigelman PM, Rantz LA. Clinical significance of Bacteroides. Arch Intern Med 1949; 84: 605-631.

4. Leach RD, Eykyn SJ, Phillips 1, Corrin B, Taylor EA. Anaerobic axillary abscess. $B M J 1979 ; 2: 5-7$.

5. Meislin HW, Lerner SA, Graves MH et al. Cutaneous abscesses: anaerobic and aerobic bacteriology and outpatient management. Ann Intern Med 1977; 7: 145-149.

6. Highet AS, Warren RE, Weekes AJ. Bacteriology and antibiotic treatment of perineal suppurative hidradenitis. Arch Dermatol 1988; 124: 1047-1051.

7. Summanen P, Baron EJ, Citron DM, Strong CA, Wexler HM, Finegold SM. Wadsworth anaerobic bacteriology manual, 5th edn. Belmont, CA, Star Publishing Company. 1993.

8. Murray PR, Baron EJ, Pfaller MA, Tenover FC, Yolken RH (eds). Manual of clinical microbiology, 6th edn. Washington, DC, ASM Press. 1995.

9. Rosebury T. Microorganisms indigenous to man. New York, McGraw-Hill. 1962.

10. Brook I, Frazier EH. Aerobic and anaerobic bacteriology of wounds and cutaneous abscesses. Arch Surg 1990; 125: 1445-1451.

11. Brook 1. Enhancement of growth of aerobic and facultative bacteria in mixed infections with Bacteroides species. Infect Immun 1985; 50: 929-931.

12. Altemeir WA. The pathogenicity of the bacteria of appendicitis peritonitis. An experimental study. Surgery 1942; 11: 374-384.

13. Brook I, Hunter V, Walker RI. Synergistic effect of Bacteroides, Clostridium, Fusobacterium, anaerobic cocci and aerobic bacteria on mortality and induction of subcutaneous abscesses in mice. $J$ Infect Dis 1984; 149: 924-928.

14. Tofte RW, Peterson PK, Schemling D, Bracke J, Kim Y, Quie PG. Opsonization of four Bacteroides species: role of the classical complement pathway and immunoglobulin. Infect Immun 1980; 27: 784-792.

15. Lev M, Keudell KC, Milford AF. Succinate as a growth factor for Bacteroides melaninogenicus. J Bacteriol 1971; 108: $175-178$.

16. Mergenhagen SE, Thonard JC, Scherp HW. Studies on synergistic infections. I: Experimental infections with anaerobic streptococci. J Infect Dis 1958; 103: 33-44.

17. Brook I. Pediatric anaerobic infection: diagnosis and management, 2nd edn. St Louis, MO, CV Mosby. 1989.

18. Finegold SM. Anaerobic bacteria in human disease. New York, Academic Press. 1977. 